\title{
Brief Report: The Impact of Social and News Media Coverage on the Dissemination of Autism Research
}

\author{
Anne Longo ${ }^{1} \cdot$ Brittany N. Hand ${ }^{1}$ (1) \\ Accepted: 22 January 2022 / Published online: 7 February 2022 \\ (c) The Author(s), under exclusive licence to Springer Science+Business Media, LLC, part of Springer Nature 2022
}

\begin{abstract}
We examined how sharing autism research articles via social and news media was associated with citations and downloads. We included articles published in 2019 from three autism-focused journals. Every 10 Twitter shares yielded a $4.4 \%$ increase in article downloads and 5.2\% increase in citations. Articles with at least one Facebook post had 23.3\% more downloads than those without. Articles with at least one news story had 56.9\% more downloads and 39.3\% more citations than those without. Descriptive analysis indicated the most shared, downloaded, and cited articles focused largely on treatments or interventions. Autism researchers should continue sharing articles via Twitter and news media because it increases the reach of their work and may better engage research and autism community members.
\end{abstract}

Keywords Autism $\cdot$ Autism spectrum disorder $\cdot$ Research dissemination $\cdot$ Social media $\cdot$ News media

\section{Introduction}

There has been an increasing push for broader communication of scientific work over the last few decades. Moving beyond poster sessions and conferences, many researchers understand that their findings are of interests to the communities involved in or affected by their work. Evidence shows that one of these highly active and interested groups is the autism community. Autism researchers may capitalize on the popularity of social media by sharing their work to generate discussion and obtain feedback from fellow researchers and autism community members. Posts on social media sites can also lead to increased news media coverage, which can allow research findings to be spread more widely (Jang et al., 2019). This dissemination can also be important because sharing research findings with the autism community increases the translation of findings into real world practice and knowledge, which makes the gap between research and implementation smaller (Clark \& Adams, 2020). However, the extent to which these efforts to communicate research more broadly have led to measurable gains in historically

Brittany N. Hand

hand.58@osu.edu

School of Health and Rehabilitation Sciences, The Ohio

State University, 453 W 10th Ave, 228E Atwell Hall,

Columbus, OH 43210, USA prioritized metrics such as article downloads and citations remains unclear.

Among articles in other content areas such as plastic surgery and substance abuse, the association between social media shares and article downloads and citations has been shown to be modest (Asaad et al., 2020; Palamar \& Strain, 2021). In comparison, news media coverage has been shown to be more highly associated with the number of citations (Anderson et al., 2020). To date, however, no studies have examined the effects of social media shares and news coverage on autism research article downloads and citations.

Thus, our objective was to determine how social media shares and news coverage are associated with research article downloads and citations among manuscripts published in three autism-focused journals: Autism, Journal of Autism and Developmental Disorders (JADD), and Autism Research. We hypothesized that the number of social media shares and news stories would be significantly associated with the number of article downloads and citations. Ultimately, this may advocate for the inclusion of a broader range of metrics for autism researchers to demonstrate the reach of their work. We also sought to describe characteristics of articles that were the most downloaded, cited, shared on social media, or covered in the media. 


\section{Methods}

\section{Article Sources}

We included manuscripts published in Autism, the Journal of Autism and Developmental Disorders (JADD), and Autism Research. We chose these three journals because they have higher impact factors and more autism-specific published papers than other journals. We also chose to include three journals instead of one so that we could have more complete data surrounding downloads and citations for autism-specific papers.

\section{Article Selection}

We included articles published in 2019 to ensure that there was adequate time for work from each month throughout the year to be cited and downloaded. We did not include articles published in 2020, as our findings may have been confounded by the effects of the Covid-19 pandemic on the research publishing process (Harrop et al., 2021). We limited the manuscripts analyzed to original articles, review articles, and short reports. Articles that were excluded from analyses included letters to the editor, commentary, and corrections. In total, there were 729 articles included, with 182 articles from Autism, 401 from JADD, and 146 from Autism Research.

\section{Data Extraction}

The first author extracted data in June 2021 and entered it into a REDCap database. We obtained the following information from each journal's website: (1) authorship; (2) initial online publication date; (3) official publication date; (4) open access status; and (5) article type (i.e., short report, original article, review article). The first author also noted each article's primary methodology based on the abstract (i.e., quantitative, qualitative, mixed method, review paper). We extracted information on the number of public tweets on Twitter, public posts on Facebook, as well as the number of stories from news sources from Altmetric.com. News stories are detected by Altmetric.com if they are posted online and contain a direct link to the article or necessary information for text mining. Autism and JADD websites report the number of downloads per article. As this information is not provided on the Autism Research website, download information was provided by the journal's editorial team. Citation information for each article was obtained from Google Scholar.

To describe the articles that were most downloaded, cited, and shared on social media, we classified articles into the Interagency Autism Coordinating Committee (IACC)'s Strategic Plan categories of: (1) Diagnosis/Screening, (2) Biology, (3) Risk Factors, (4) Treatments/Interventions, (5) Services, (6) Lifespan Issues, and (7) Surveillance/Infrastructure (IACC, 2018). We also recorded the populations that these articles studied.

\section{Analyses}

We calculated descriptive statistics, including frequencies and percentages, medians, and interquartile ranges. Our dependent variables were: (1) number of downloads; and (2) number of citations. Our primary independent variables were social media and news coverage, quantified by: (1) Twitter shares, (2) Facebook posts, and (3) news stories. We used a generalized linear model with a gamma distribution and $\log$ link to quantify the relationship between our dependent and independent variables, in accordance with standards for analyzing continuous, positively-skewed, non-negative data (Ng \& Cribbie, 2017). We also classified articles in the 90th percentile for downloads as "highly downloaded" and articles in the 90th percentile for citations as "highly cited." We used multivariable logistic regression to quantify the relative contributions of each independent variable to the odds being highly downloaded or highly cited. All multivariable analyses controlled for open access status, the number of months since initial online publication (i.e., e-publication ahead of print), and journal of origin. Relationships between the independent and dependent variables were considered statistically significant if the $95 \%$ confidence interval for the parameter estimate did not cross 1.0 .

\section{Institutional Review Board Approval}

This analysis is not based on human subjects research so it was exempt from review from the authors' institutional review boards.

\section{Results}

A description of included articles is shown in Table 1. Most articles used quantitative methods (82\%) and were full-length (84\%). Overall, $18 \%$ of the articles were open access. The three journals together had a median of 1110 downloads and 9 citations per article. $26 \%$ of the articles has been shared on a public wall on Facebook, and the median number of Tweets per article was 8 .

Our multivariable statistical models indicated every 10 Twitter shares yielded, on average, a $4.4 \%$ increase in the number of downloads and $5.2 \%$ increase in citations (Table 2). Articles with at least one Facebook post had $23.3 \%$ more downloads but did not significantly differ in 
Table 1 Description of included articles by journal

\begin{tabular}{|c|c|c|c|c|}
\hline & Autism $(\mathrm{N}=182)$ & Autism research $(\mathrm{N}=146)$ & $\mathrm{JADD}(\mathrm{N}=401)$ & Total $(\mathrm{N}=729)$ \\
\hline Views/downloads per article, Median (IQR) & $1365(836,2330)$ & $622(458,1257)$ & $1186(775,2001)$ & $1110(666,1971)$ \\
\hline Citations per article, Median (IQR) & $12(7,23)$ & $7(5,16)$ & $8(4,15)$ & $9(5,17)$ \\
\hline Twitter posts, Median (IQR) $)^{\mathrm{a}}$ & $19.5(5,38)$ & $8.5(3,24)$ & $5(1,15)$ & $8(2,23)$ \\
\hline Any Facebook post, N (\%) & $84(46.2)$ & $32(21.9)$ & $77(19.2)$ & $193(26.5)$ \\
\hline Any news story, N(\%) & $27(14.8)$ & $19(13.0)$ & $23(5.7)$ & $69(9.5)$ \\
\hline Open access, $\mathrm{N}(\%)$ & $40(22.0)$ & $19(13.0)$ & $73(18.2)$ & $132(18.1)$ \\
\hline \multicolumn{5}{|l|}{ Article type, N (\%) } \\
\hline Full-length article & $153(84.1)$ & $133(91.1)$ & $326(81.3)$ & $612(84.0)$ \\
\hline Review article & $15(8.2)$ & $8(5.5)$ & $25(6.2)$ & $48(6.68)$ \\
\hline Short report & $14(7.7)$ & $5(3.4)$ & $50(12.5)$ & $69(9.5)$ \\
\hline \multicolumn{5}{|l|}{ Methodology, N(\%) } \\
\hline Review or other & $20(11.0)$ & $9(6.2)$ & $27(6.7)$ & $56(7.7)$ \\
\hline Qualitative or mixed method & $40(22.0)$ & $2(1.4)$ & $35(8.7)$ & $77(10.6)$ \\
\hline Quantitative & $122(67.0)$ & $135(92.5)$ & $339(84.5)$ & $596(81.8)$ \\
\hline
\end{tabular}

${ }^{\mathrm{a}} 100 \%$ of articles were shared at least once on Twitter

Table 2 Association of social media shares and news media coverage with downloads or citations

\begin{tabular}{|c|c|c|c|c|}
\hline \multirow[b]{2}{*}{ Variable } & \multicolumn{2}{|l|}{ Downloads } & \multicolumn{2}{|l|}{ Citations } \\
\hline & $\begin{array}{l}\% \text { Increase in downloads } \\
(95 \% \mathrm{CI})^{\mathrm{a}}\end{array}$ & $\begin{array}{l}\text { Adjusted odds of high down- } \\
\text { loads }(95 \% \mathrm{CI})^{\mathrm{b}}\end{array}$ & $\begin{array}{l}\% \text { Increase in citations } \\
(95 \% \mathrm{CI})^{\mathrm{a}}\end{array}$ & $\begin{array}{l}\text { Adjusted odds of } \\
\text { high citations }(95 \% \\
\text { CI) }\end{array}$ \\
\hline Every 10 Twitter shares & $4.4(3.3,5.5)^{*}$ & $1.1(1.1,1.2)^{*}$ & $5.2(3.7,6.7)^{*}$ & $1.2(1.1,1.2)^{*}$ \\
\hline Any Facebook post & $23.3(10.6,37.6)^{*}$ & $1.2(1.1,1.4)^{*}$ & $14.9(-0.6,32.7)$ & $1.4(0.8,2.7)$ \\
\hline Any News story & $57.1(34.6,83.4) *$ & $9.5(3.7,24.6) *$ & $39.3(14.0,70.3)^{*}$ & $3.2(1.6,6.5)^{*}$ \\
\hline
\end{tabular}

${ }^{a}$ Estimates obtained via gamma regression with a log link, controlling for journal, open access status, and months since initial online publication

${ }^{b}$ Odds ratios obtained from logistic regression controlling for journal, open access status, and months since initial online publication

* Statistically significant

the number of citations from those without. Articles with at least one news story had $56.9 \%$ more downloads and $39.3 \%$ more citations than those without.

Our multivariable logistic regression models indicated that for every 10 Twitter shares, there was 1.1 times greater odds of high downloads and 1.2 times greater odds of high citations (Table 2). Articles with at least one news story had 9.5 times greater odds of high downloads and 3.2 times greater odds of high citations. Having at least one Facebook post was not significantly associated with odds of high downloads or citations.

In describing the characteristics of the five most downloaded, cited, and shared articles (See Supplemental Table), the plurality were in the IACC categories of Diagnosis/ Screening (symptomology) (28\%) or Treatments/Interventions (28\%), and most were focused on adults on the autism spectrum (60\%). About $44 \%$ were quantitative and $32 \%$ were either qualitative or mixed methods. Eighty percent were open access. All of the top five downloaded articles were classified into the IACC categories of Diagnosis/Screening or Treatments/Interventions, but the five most cited articles represented a variety of IACC categories. Among the articles shared most in social and news media, most (73\%) fit in the Services, Treatments/Interventions, or Infrastructure/ Surveillance categories.

\section{Discussion}

In the past decade, there has been more research on social media use and its effects on article dissemination, including the effects on article downloads and citations (Palamar $\&$ Strain, 2021). Our paper provides the first analysis of the relationship between autism research dissemination on social and news media and downloads and citations. Even after controlling for open access, which is known to greatly increase the chance of article downloads and citations, we found that social media shares and news media coverage 
were significantly associated with more article downloads and citations. Specifically, our analyses showed that article downloads increased significantly with every 10 Twitter shares, at least one Facebook share, and any news media coverage (Table 2). Articles citations increased significantly with every 10 Twitter shares and any news media coverage.

Our findings also provide the first description, to our knowledge, of characteristics of autism research articles with most downloads, citations, social media shares, and news coverage. Interestingly, despite being the largest area of funding for autism research, studies on the biology of autism were not among the most downloaded, cited, or shared articles (Harris et al., 2021). In contrast, most of the highly downloaded, cited and shared articles focused on high-priority research areas identified by the IACC like treatments and interventions and services (IACC, 2018).

Autism is a popular topic of online discussion among researchers and the autism community. The autism community is incredibly active on social media, as autistic individuals spend more time using the internet and computermediated communication than non-autistic individuals (Kim, 2019; MacMullin et al., 2015). Widespread sharing of an article increases visibility among autism community members who, if interested, may download the article to learn more. Additionally, sharing an article via social media allows for engagement and opportunity for discussion with the autism community and researchers. Articles in this study tended to be shared most on Twitter, which is not surprising as it is the most used researcher posting cite (Dol et al., 2019) and is used by the autism community more than Facebook (van Schalkwyk et al., 2017).

Our results showed that having news media cover a research article had a large positive association with downloads and citations. However, relatively few articles in our study $(<10 \%)$ were covered in news media. The five articles that were most covered in news media (Supplemental Table) tended to be open access (80\%), focus on autistic children and adolescents (100\%), and cover topics like: (a) outcomes as a function of race or ethnicity (40\%), or (b) novel treatments and interventions (40\%). This result shows that despite increased focus on social media, news media still plays an important role in widespread reach of research findings and provides insights as to the types of articles most likely to receive media coverage.

\section{Limitations}

Given that data from the journal websites, Almetric.com, and Google Scholar, including information on downloads, citations, and social media shares, updates daily, double data entry was not possible. Almetric.com limits their information on social media shares to public posts; posts shared on private Twitter or Facebook pages would not be captured in our data. Almetric.com also does not include social media posts from other popular platforms, such as YouTube and Instagram, so our data may underestimate total social media shares. Our data are also limited to the three journals we studied. We chose to focus on three high-impact, credible journals that publish mostly or exclusively articles about autism. However, we recognize that there are other autism journals, with different focuses and audiences, so our results may not be generalizable in other journals. We also acknowledge there are other ways of sharing research, such as through oral and poster presentations at conferences, that were beyond the scope of this study but could be considered in future work.

\section{Conclusion}

The dissemination of autism research via social and news media is associated with more downloads and citations. Our results add to studies from other content areas, while providing the first examination of the relationship between media and article reach in autism research. Sharing information via media may be a way of quantifying the impact of an article more quickly, while traditional indicators, like citations, may take more time to accumulate due to lengthy peer-review and publication processes. Autism research articles should continue to be posted and shared online (i.e., Facebook, Twitter, and in news media) as it is linked with higher dissemination and allows for engagement and discussion with the autism community.

Supplementary Information The online version contains supplementary material available at https://doi.org/10.1007/s10803-022-05464-8.

Acknowledgments The project described was supported by Award Number Grant UL1TR002733 from the National Center For Advancing Translational Sciences. The content is solely the responsibility of the authors and does not necessarily represent the official views of the National Center For Advancing Translational Sciences or the National Institutes of Health.

Author contribution $\mathrm{AL}$ and $\mathrm{BH}$ contributed to study conception and design. AL extracted data from the three journals. BH performed all data analyses. AL and $\mathrm{BH}$ jointly drafted and iteratively revised this manuscript.

\section{Declarations}

Conflict of interest The authors declare no conflicts of interest.

Ethical Approval This analysis is not based on human subjects research so it was exempt from review from the authors' institutional review boards. 


\section{References}

Anderson, P., Odom, A., Gray, H., Jones, J., Christensen, W., Hollingshead, T., Hadfield, J., Evans-Pickett, A., Frost, M., Wilson, C., Davidson, L., \& Seeley, M. (2020). A case study exploring associations between popular media attention of scientific research and scientific citations. PLOS ONE, 15(7), e0234912. https://doi. org/10.1371/journal.pone.0234912

Asaad, M., Howell, S. M., Rajesh, A., Meaike, J., \& Tran, N. V. (2020). Altmetrics in plastic surgery journals: Does it correlate with citation count? Aesthetic Surgery Journal, 40(11), 628-635. https:// doi.org/10.1093/asj/sjaa158

Clark, M., \& Adams, D. (2020). Listening to parents to understand their priorities for autism research. PLoS ONE, 15(8), e0237376. https://doi.org/10.1371/journal.pone.0237376

Dol, J., Tutelman, P., Chambers, C., Barwick, M., Drake, E., Parker, J., Parker, R., Benchimol, E., George, R. B., \& WItteman, H. (2019). Health Researchers' use of social media: scoping review. Journal of Medical Internet Research, 21(11), e13687.

Harris, L., Gilmore, D., Longo, A., \& Hand, B. (2021). Short report: Patterns of US federal autism research funding during 2017-2019. Autism. https://doi.org/10.1177/13623613211003430

Harrop, C., Bal, V., Carpenter, K., \& Halladay, A. (2021). A lost generation? The impact of the COVID-19 pandemic on early career ASD researchers. Autism Research, 14(6), 1078-1087. https://doi. org/10.1002/aur.2503

Interagency Autism Coordinating Committee. (2018). IACC strategic plan for autism spectrum disorder 2018-2019. Retrieved from https://iacc.hhs.gov/publications/strategic-plan/2019/

Jang, S. M., Mckeever, B. W., Mckeever, R., \& Kim, J. K. (2019). From social media to mainstream news: The information flow of the vaccine-autism controversy in the US, Canada, and the UK. Health Communication, 34(1), 110-117. https://doi.org/10.1080/ 10410236.2017.1384433

Kim, S. Y. (2019). The experiences of adults with autism spectrum disorder: Self-determination and quality of life. Research in Autism Spectrum Disorders, 60, 1-15. https://doi.org/10.1016/j.rasd. 2018.12.002

MacMullin, J., Lunsky, Y., \& Weiss, J. (2015). Plugged in: Electronics use in youth and young adults with autism spectrum disorder. Autism, 20(1), 45-54. https://doi.org/10.1177/1362361314566047

Ng, V. K. Y., \& Cribbie, R. A. (2017). Using the gamma generalized linear model for modeling continuous, skewed and heteroscedastic outcomes in psychology. Current Psychology, 36(2), 225-235. https://doi.org/10.1007/s12144-015-9404-0

Palamar, J. J., \& Strain, E. C. (2021). News and social media coverage is associated with more downloads and citations of manuscripts that focus on substance use. Drug and Alcohol Dependence, 218, 108357. https://doi.org/10.1016/j.drugalcdep.2020.108357

van Schalkwyk, G. I., Marin, C. E., Ortiz, M., Rolison, M., Qayyum, Z., McPartland, J. C., Lebowitz, E. R., Volkmar, F. R., \& Silverman, W. K. (2017). Social media use, friendship quality, and the moderating role of anxiety in adolescents with autism spectrum disorder. Journal of Autism and Developmental Disorders, 47(9), 2805-2813. https://doi.org/10.1007/s10803-017-3201-6

Publisher's Note Springer Nature remains neutral with regard to jurisdictional claims in published maps and institutional affiliations. 